\title{
NÍVEL DE ATIVIDADE FÍSICA E ACÚMULO DE TEMPO SENTADO EM ESTUDANTES DE MEDICINA
}

\author{
PHYSICAL ACTIVITY LEVEL AND ACCUMULATION OF SITTING TIME AMONG \\ MEDICAL STUDENTS \\ NIVEL DE ACTIVIDAD FÍSICA Y LA ACUMULACIÓN DE TIEMPO SENTADO \\ EN ESTUDIANTES DE MEDICINA
}

ARTIGO ORIGINAL

\begin{abstract}
Leandro Luiz de Oliveira Raddi (Educador físico)

João Pedro da Silva Júnior

(Educador físico)

Gerson Luis de Moraes Ferrari

(Educador físico)

Luís Carlos de Oliveira'

(Educador físico)

Victor Keihan Rodrigues Matsudo' (Médico)

1. Centro de Estudos do Laboratório de Aptidão Física de São Caetano do Sul (CELAFISCS)
\end{abstract}

\section{Correspondência:}

Rua Heloisa Pamplona, 269, Bairro Fundação, São Caetano do Sul, SP, Brasil - 09520-320.

celafiscs@celafiscs.org.br

\begin{abstract}
RESUMO
Objetivo: Verificar, no período de dez anos, o comportamento do nível de atividade física e do acúmulo de tempo sentado em estudantes de Medicina. Métodos: A amostra foi composta por 186 estudantes do terceiro ano em 2000-2001 (grupo I) e em 2011 (grupo II) com média de idade de 21,23 1,38 anos. Os dados do nível de atividade física e do tempo sentado foram coletados através do questionário IPAQ (versões curta e longa). Os questionários foram aplicados individualmente e foram autopreenchidos. Análise estatística: Os resultados foram descritos através de frequência e porcentagem do nível de atividade física e média e desvio padrão para o acúmulo de tempo sentado. A comparação foi feita através do teste do qui-quadrado e do teste $t$ para amostras independentes, sendo o nível de significância adotado de $p<0,05$. Resultados: No período de dez anos foi encontrada uma diminuição acentuada e significante de $27,4 \%$ entre os indivíduos que atingiram as recomendações no grupo I (75,2\%) em relação aos que atingiram no grupo II (47,8\%). O acúmulo de tempo sentado não apresentou diferenças significantes, sendo as médias encontradas de $=8,92 \mathrm{e}=8,72$ horas para dias de semana e de $=7,27$ e $=7,70$ horas para dias do fim de semana. Conclusão: Após dez anos, os estudantes de medicina apresentaram menor nível de atividade física sendo que não houve mudança no acúmulo de tempo sentado.
\end{abstract}

Palavras chave: estudantes de medicina, atividade física, sedentarismo.

\begin{abstract}
Objective: To determine, within ten years the physical activity level and accumulation of sitting time among medical students. Methods: The sample consisted of 186 third year students of Medical School in 2000-2001 (Group I) and 2011 (Group II), with a mean age of 21.23 1.38. The data on the level of physical activity and sitting time were collected through questionnaire IPAQ (short and long versions). The questionnaires were administered individually and were self-completed. Statistical analysis: The results were reported as frequency and percentage for the level of physical activity and mean and standard deviation for the accumulation of time sitting. The comparison was made using the chi-square and t test for independent samples, and the significance level was $p<.05$. Results: In the ten-year period a marked and significant decrease of $27.4 \%$ was found among subjects who met the recommendations in Group I (75.2\%) compared to those achieved in group II (47.8\%). Sitting time showed no significant differences, and means were $=8.92$ and $=8.72$ hours for weekdays and $=7.27$ and $=7.70$ hours for the weekend days. Conclusion: After ten years, the medical students had lower levels of physical activity with no change in the accumulation of sitting time.
\end{abstract}

Keywords: students medical, motor activity, sedentary lifestyle.

\section{RESUMEN}

Objetivo: Verificar, en el período de diez años, el comportamiento del nivel de actividad física y de la acumulación de tiempo sentados en estudiantes de Medicina. Métodos: la muestra se compuso de 186 estudiantes del tercer año en 2000-2001 (grupo I) y en 2011 (grupo II), con promedio de edad de 21,23 1,38 años. Los datos sobre nivel de actividad física y de tiempo sentado se recolectaron mediante el cuestionario IPAQ (versiones corta y larga). Los cuestionarios fueron aplicados individualmente y se completaron por los mismos estudiantes. Análisis estadístico: Los resultados se describieron mediante frecuencia y porcentaje del nivel de actividad física, y promedio y desviación estándar para la acumulación de tiempo sentado. La comparación se hizo por medio de la prueba de chi-cuadrado y de la prueba t para muestras independientes, siendo el nivel de significancia adoptado de $p<0,05$. Resultados: en el período de diez años se encontró una disminución acentuada y significativa de 27,4\% entre los individuos que alcanzaron las recomendaciones en el grupo I (75,2\%) en comparación con quienes las consiguieron en el grupo II (47,8\%). La acumulación de tiempo sentado no presentó diferencias significativas, siendo los promedios hallados de $=8,92 y=8,72$ horas para días laborales de la semana y de $=7,27$ y $=7,70$ horas para días de fines de semana. Conclusión: Después de diez años, los estudiantes de Medicina presentaron menor nivel de actividad física, siendo que no hubo cambio en la acumulación del tiempo sentado.

Palabras clave: estudiantes de medicina, actividad motora, estilo de vida sedentario. 


\section{INTRODUÇÃO}

O termo prevenção da saúde é definido como intervenções orientadas para evitar o surgimento ou evolução de doenças específicas nas populações ${ }^{1,2}$. Alguns estudos tem sido feitos e estimulados em várias áreas do conhecimento humano nas últimas décadas em relação à prevenção da saúde ${ }^{2-4}$. A atividade física é definida como qualquer movimento corporal produzido pelos músculos esqueléticos que resulta em gasto energético maior do que os níveis de repouso ${ }^{5}$. Conforme o posicionamento da Sociedade Brasileira de Medicina e Esporte ${ }^{6}$ a saúde do homem pode ser preservada e aprimorada pela prática regular de atividade física. Estudos epidemiológicos vêm demonstrando expressiva associação entre estilo de vida ativo, menor risco de morte, e melhor qualidade de vida, além da atividade física ser uma importante forma de prevenção no desenvolvimento das doenças crônico-degenerativas ${ }^{4,7,8}$.

As recomendações atuais de atividade física para adultos segundo o Colégio Americano de Medicina e Esporte e a Associação Americana do Coração ${ }^{9}$ consiste na frequência mínima de cinco vezes por semana, com duração de pelo menos trinta minutos por dia, de modo contínuo ou acumulado em intensidade moderada, ou de pelo menos três vezes por semana, por vinte minutos ao dia de modo contínuo na intensidade vigorosa, ou ainda, as duas intensidades podem ser combinadas para atingir às recomendações.

O comportamento sedentário é qualquer atividade que reduza o gasto corporal energético a valores próximos ao de repouso, o que incluí atividades como sentar, assistir televisão, usar o computador ou dormir, sendo o acúmulo de tempo sentado um indicativo deste ${ }^{10,11} \mathrm{Em}$ um estudo de Katzmarzyk et al. ${ }^{12}$ foi demonstrado que quanto maior o acúmulo de tempo sentado maior é a taxa de mortalidade por todas as causas, e para alguns autores há a necessidade de avaliar tanto a exposição ao baixo nível de atividade física quanto a comportamentos sedentários, pois existem evidências sugerindo que estes são independentes e têm diferentes efeitos sobre a saúde ${ }^{10,11}$.

Matsudo e Andrade ${ }^{13}$ consideram que entre os fatores tidos como motivadores da adoção de um estilo de vida mais ativo estão o incentivo de amigos, a ação de programas específicos e o aconselhamento dos profissionais da área da saúde, sendo que este último é considerado bastante efetivo especialmente na população mais idosa. 0 comportamento saudável pode ser estimulado com intervenções e o médico deve contribuir para isto, pois além de ser o profissional da área da saúde mais influente ele é elemento fundamental para promover e prevenir saúde e não somente tratando doenças ${ }^{13-18}$. Assim, considerando o modelo de comportamento do médico como reforço no impacto da prescrição, o objetivo do presente estudo foi verificar o comportamento do nível de atividade física e do acúmulo de tempo sentado em estudantes de medicina em um período de dez anos.

\section{MATERIAIS E MÉTODOS}

A amostra foi composta por estudantes do terceiro ano da Faculdade de Ciências Médicas da Santa Casa de São Paulo (FCMSCSP) (N=186, idade $=21,23+1,38$ ), divididos em dois grupos 2000-2001 (grupo I) e 2011 (grupo II). A escolha da amostra foi feita por conveniência e o projeto de pesquisa foi aprovado pelo Comitê de Ética em Pesquisa de São Caetano do Sul.

O instrumento utilizado para medida do nível de atividade física foi o Questionário Internacional de Atividade Física (IPAQ), em suas versões curta e longa. Este teve sua validade testada em ambas as versões no Brasil por Matsudo, et al. ${ }^{19}$ e Pardini, et al. ${ }^{20}$. Dentre as várias conclusões observadas, o IPAQ em suas duas formas teve sua validade e reprodutibilidade comparadas com as de outros instrumentos já aceitos e utilizados internacionalmente para medir nível de atividade física. Não foram observadas diferenças estatisticamente significantes nos resultados obtidos através das duas versões quando estes foram comparados.

As perguntas do questionário estão relacionadas às atividades realizadas na semana anterior à aplicação do questionário. Os estudantes de medicina tiveram seus dados tabulados e a variável nível de atividade física foi categorizada naqueles que atingiam às recomendações e os que não atingiam às recomendações, conforme as guias estabelecidas ${ }^{9,21}$. Em relação ao tempo sentado, os estudantes foram questionados sobre quanto tempo gastavam sentados em um dia de semana e em um dia de fim de semana na última semana em duas perguntas que também fazem parte do IPAQ.

O questionário foi entregue aos sujeitos da pesquisa, que receberam então a mesma orientação verbal. Uma orientação escrita sobre os procedimentos adequados também estava presente no próprio questionário. Os questionários foram aplicados individualmente e foram autopreenchidos, sendo que qualquer dúvida era esclarecida na hora do preenchimento pelos responsáveis treinados para a aplicação do $I P A Q$, com os sujeitos do estudo não se comunicando entre si.

\section{Estatísticas}

Os resultados foram descritos através de frequência e porcentagem para o nível de atividade física e em média e desvio padrão para o acúmulo de tempo sentado. A análise estatística feita foi o teste do qui-quadrado para a comparação das porcentagens e "teste $t^{\prime \prime}$ para amostras independentes para a comparação das médias. Todos os cálculos foram feitos utilizando o Statistical Package for the Social Sciences (SPSS) versão 18.0 e o nível de significância adotado foi de $p<0,05$.

\section{RESULTADOS}

A tabela 1 mostra o total da amostra, $\mathrm{N}=186 \mathrm{com}$ idade média de 21,23 anos, dos quais 109 eram do sexo masculino e 77 do feminino. O Grupo I era formado por 117 estudantes de medicina com idade média de 20,99 anos (78 do sexo masculino e 39 do sexo feminino) e o Grupo II era composto por 69 indivíduos com idade medida de 21,76 anos (31 do sexo masculino e 38 do sexo feminino).

Em relação à comparação entre os estudantes de medicina da FCMSCSP que atingem as recomendações de atividade física, (figura 1) observa-se que no grupo I 75,2\% dos sujeitos atingiram as recomendações, enquanto que no grupo II apenas 47,9\% dos sujeitos as atingiram, havendo uma diminuição acentuada e significativa de $27,4 \%(p<0,05)$

Para a variável acúmulo de tempo sentado dos estudantes de medicina da FCMSCSP (figura 2) não houve diferença significativa quando comparados os dois grupos $(p<0,05)$, sendo as médias encontradas de $=8,92$ e $=8,72$ horas para dias de semana e de $=7,27$ e de $=7,70$ horas para dias do fim de semana.

Tabela 1. Caracterização dos estudantes de Medicina.

\begin{tabular}{|c|c|c|c|c|c|}
\hline Grupo & $\mathrm{N}$ & Masculino & Feminino & $\begin{array}{l}\text { Idade média } \\
\text { (Anos) }\end{array}$ & $S D^{*}( \pm)$ \\
\hline Total & 186 & 109 & 77 & 21,23 & 1,38 \\
\hline Grupo I** & 117 & 78 & 39 & 20,99 & 1,60 \\
\hline Grupo $\|^{* * *}$ & 69 & 31 & 38 & 21,76 & 1,06 \\
\hline
\end{tabular}

\section{DISCUSSÃO}

Os resultados do presente estudo mostraram que no período de dez anos foi encontrada uma diminuição acentuada e significante de $27,4 \%$ entre os indivíduos que atingiram as recomendações de atividade física no grupo I $(75,2 \%)$ em relação aos que as atingiram 


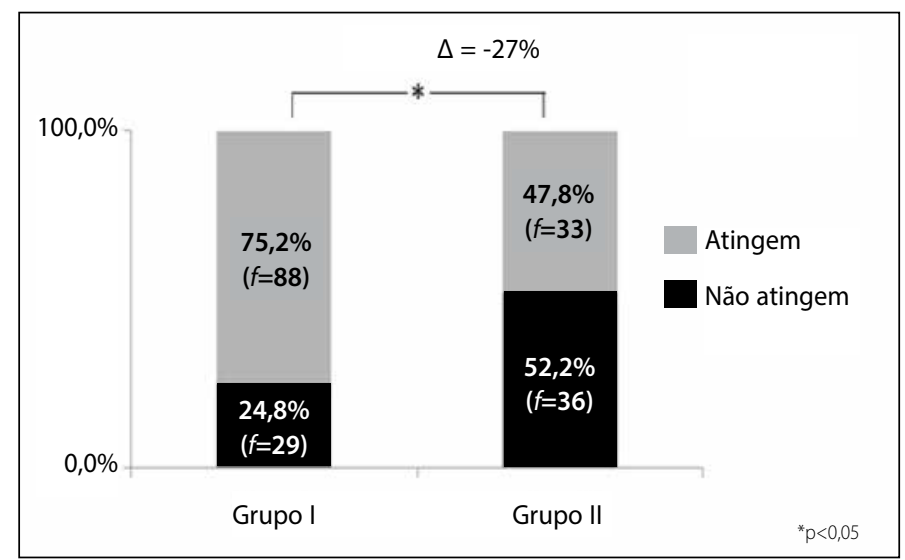

Figura 1. Porcentagem dos estudantes de Medicina que atingiram as recomendações de atividade física.

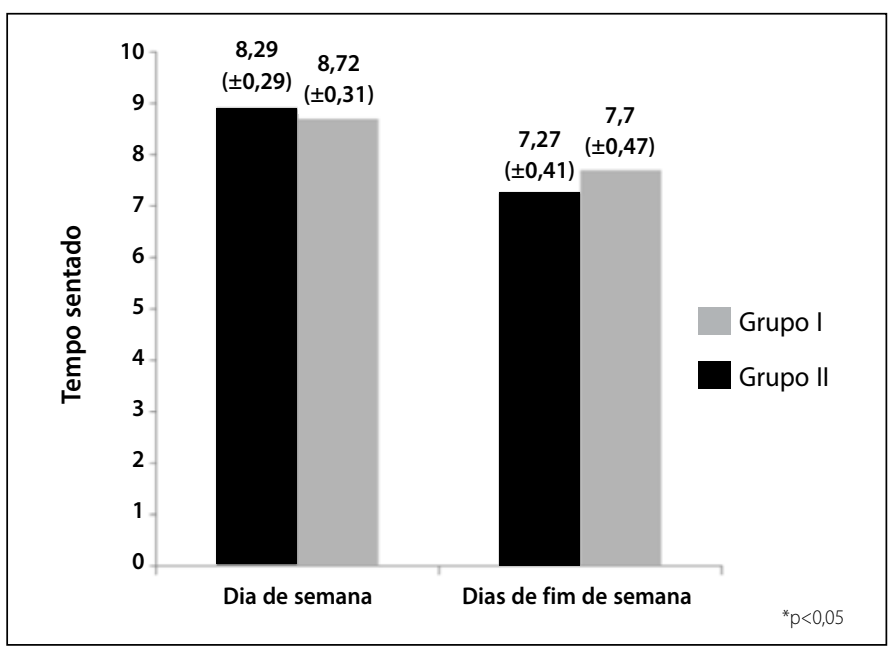

Figura 2. Acúmulo de tempo sentado dos estudantes de Medicina que atingiam as recomendações de atividade física.

no grupo II (47,8\%). O acúmulo de tempo sentado não apresentou diferenças significantes, sendo as médias encontradas de $=8,92 \mathrm{e}$ de $=8,72$ horas para dias de semana de $=7,27$ e de $=7,70$ horas para dias do fim de semana.

Os achados do presente estudo corroboram os achados de Marcondelli et al.22 que avaliaram 281 estudantes universitários da área da saúde (medicina, enfermagem, nutrição, farmácia, odontologia e educação física) de uma faculdade de Brasília. Os autores observaram o alto nível de sedentarismo e de baixa atividade física, sendo o curso de educação física o único a mostrar-se mais ativo e o curso de medicina o segundo curso a ter mais estudantes sedentários ou com baixo nível de atividade física.

Em um estudo ${ }^{23}$ feito com 87 universitários de diversas áreas do conhecimento em uma faculdade do Tocantins, os autores não encontraram diferença significativa no que se refere ao nível de atividade física entre as áreas. Fontes e Viana ${ }^{24}$ também constataram em 1503 estudantes de uma faculdade da Paraíba maior prevalência de indivíduos que alcançavam as recomendações havendo diferenças significativas relacionadas ao curso, turno das aulas, dentre outros indicativos, também foi verificada uma tendência de o nível de atividade física decrescer conforme os universitários avançavam nos anos de sua graduação. Silva et al. ${ }^{25}$ analisando 280 universitários da área da saúde (farmácia, bioquímica, odontologia, ciências biológicas e educação física) de uma faculdade de Minas Gerais constatou que $75,4 \%$ alcançavam as recomendações de atividade física, não havendo também diferenças significativa entre as áreas, contudo a área da educação física novamente demonstrou maior porcentagem de indivíduos fisicamente ativos (90\%).

A literatura tem mostrado que há uma consciência crescente de que o bem-estar dos médicos e seus hábitos afetam a saúde de seus pacientes, o que também incluí a atividade física ${ }^{16,17,26-28}$. Um estudo prospectivo ${ }^{16} \mathrm{com}$ uma amostra representativa $(\mathrm{N}=2.316)$ dos estudantes de medicina dos EUA de 16 universidades evidenciou que os estudantes de medicina que cumpriram ou aderiram às recomendações de atividade física ao longo dos quatro anos do estudo demonstraram melhor saúde mental, maior taxa de prescrição da atividade física e maior influência dessas prescrições em relação aos seus pacientes. Em um estudo transversal ${ }^{17}$ feito com 661 estudantes de medicina colombianos do primeiro e do quinto ano de medicina foram analisados os hábitos de vida e foram encontradas associações positivas entre aqueles que mantinham hábitos saudáveis e a saúde de seus pacientes, além dos estudantes apresentarem maior prática do aconselhamento, o que reforça a afirmação médico saudável igual a paciente saudável.

Segundo Lobelo et al. ${ }^{27}$ os médicos são uma fonte respeitável de informações relacionadas à saúde e podem fornecer um feedback contínuo de aconselhamento preventivo e de acompanhamento aos pacientes. Contudo, várias barreiras para o aconselhamento existem, incluindo a formação insuficiente, a falta de motivação, de aperfeiçoamento e os hábitos pessoais.

No presente estudo, podemos observar que apenas 47,8\% da amostra estudada cumpriu as recomendações de atividade física e que a média de acúmulo do tempo sentado para os dias de semana e de fim de semana foi de aproximadamente 8 e 9 horas, respectivamente, o que corrobora outros estudos realizados pelo mesmo grupo de pesquisadores ${ }^{18,29}$. Estes evidenciaram que possivelmente os estudantes de medicina e os médicos brasileiros não estejam preparados para prescrever a atividade física, pois a maior parte dos sujeitos que fizeram parte desses levantamentos desconhecia os parâmetros das recomendações (frequência, duração, modo e intensidade) ${ }^{9}$ Em outro estudo do Celafiscs ${ }^{15}$ o nível de conhecimento dos parâmetros das recomendações de atividade física dos estudantes de medicina do primeiro e segundo ano de uma faculdade de São Paulo foi insatisfatório. Foi observado em outra pesquisa ${ }^{14}$ que o mesmo ocorreu quando analisaram estudantes do sexto ano de medicina da mesma instituição. Da mesma forma, a maior parte dos médicos do serviço público pesquisados pelo Celafiscs ${ }^{29}$ não conheciam as recomendações. Um estudo realizado com ortopedistas brasileiros ${ }^{18}$ demonstrou que apenas 8,8\% da amostra conhecia todos os parâmetros de recomendação de atividade física, embora 99,2\% dos mesmos ortopedistas terem relatado prescrever a atividade física a seus pacientes.

Baseado nos achados citados anteriormente, o Exercise is Medicine (EIM), lançado pelo American College of Sports Medicine e apoiado pelo Centro de Estudos do Laboratório de Aptidão Física de São Caetano do Sul, tem desenvolvido uma iniciativa para encorajar os médicos da atenção primária e outros prestadores de cuidados de saúde a incluir o exercício na vida diária e na elaboração de planos de tratamento para os pacientes. O EIM enfatiza a necessidade de uma avaliação regular de exercício e atividade física ao longo de serviços médicos como parte integrante da prevenção e tratamento da doença ${ }^{30}$.

Dubnov-Raz, Berry e Constantini26 apontaram que um estilo de vida saudável é a base da medicina preventiva e os profissionais da saúde podem desconsiderar manter uma dieta saudável e o hábito da atividade física talvez pela sensação de proteção devido ao seu conhecimento específico. Van der Veer et al, ${ }^{28}$ baseados em uma amostra de 962 estudantes de medicina holandeses, verificaram a prevalência de 
comportamentos não saudáveis, sendo que o estudo concluíu que as faculdades de medicina necessitam abordar formas de promover comportamentos saudáveis tanto para o benefício de seus alunos, como dos médicos e dos pacientes.

Assim, vários estudos ${ }^{16,17,26-28}$ concordam com a necessidade de estudar o nível de atividade física e comportamentos relacionados à saúde dos estudantes de medicina, sendo importante a descrição destes aspectos do grupo, pois poderão servir para elaborar estratégias e intervenções como medida preventiva para a adoção de hábitos de vida saudáveis e a melhoria da qualidade de vida tantos dos estudantes quanto de seus pacientes.

Uma possível limitação deste estudo foi a análise de ambos os sexos concomitantemente. Em Marcondelli et al. ${ }^{22}$ houve diferença significante entre o nível de atividade física entre homens mulheres. Já em Rodrigues et al. ${ }^{23}$ embora não tenham encontrado esta diferença, observaram que houve tendência dos homens serem mais fisicamente ativos que as mulheres, enquanto Silva et al. ${ }^{25}$ analisando somente o curso de educação física e Fontes e Viana ${ }^{24}$ não encontraram diferença entre os sexos. Outra provável limitação foi o uso das versões curta e longa do Questionário Internacional de Atividade Física (IPAQ), embora, dois estudos nacionais que testaram ambas as versõe $e^{19,29}$ evidenciaram que não há diferenças estatisticamente significativas entre os dois instrumentos para a análise do nível atividade física.

O conjunto de evidências permite afirmar que médicos e estudantes de medicina que cumprem as recomendações de atividade física possuem melhor saúde mental, prescrevem mais a atividade física e estas têm maior influência sobre seus pacientes. Sendo assim, é importante haver intervenções sobre o comportamento dos estudantes de medicina para que estes cumpram as recomendações de atividade física, considerando gênero, ano de graduação e região em que estudam.

\section{CONCLUSÃO}

Após dez anos os estudantes de Medicina da FCMSCSP do grupo II (2011) apresentaram pior nível de atividade física em relação aos do grupo I (2000-2001), sendo que não houve mudança no acúmulo de tempo sentado.

Todos os autores declararam não haver qualquer potencial conflito de interesses referente a este artigo.

\section{REFERÊNCIAS}

1. Agência Nacional de Saúde Suplementar (Brasil). Promoção da saúde e prevenção de riscos e doenças na saúde suplementar: Manual técnico / agência nacional se saúde suplementar. - $2^{\circ}$ ed. rev. e atual. - Rio de Janeiro: ANS, 2007:15-7.

2. Czeresnia D. O conceito de saúde e a diferença entre prevenção e promoção. Cad Saúde Pública.1999;15(4):701-9.

3. Arouca ASS. O dilema preventivista contribuição para a compreensão crítica da medicina preventiva. Tese de Doutoramento apresentada à Faculdade de Ciências Médicas da Universidade Estadual de Campinas: 1975.

4. Hallal PC, Dumith SC, Bastos JP, Reichert, FF, Siqueira FV e Azevedo MR. Evolução da pesquisa epidemiológica em atividade física no Brasil: Revisão sistemática. Rev Saúde Pública. 2007;41(3):453-60.

5. Caspersen CJ, Powell KE and Christenson GM. Physical activity, exercise, and physical fitness: definitions and distinctions for health-related research. Public Health Reports. 1985;(100) 2:126-31

6. Carvalho T, Nóbrega ACL, Lazzoli JK, Magni JRT, Rezende L, Drummond FA, Oliveira MAB, De Rose EH, Araújo CGS e Teixeira JAC. Posição oficial da sociedade brasileira de medicina do esporte: Atividade física e saúde. Rev Bras Med Esporte. 1999;(2) 4:79-81.

7. Aquino EML, Barreto SM, Bensenor IM, Carvalho MS, Chor D, Duncan BB, Lotufo PA, Mill JG, Molina MDC, Mota ELA, Passos VMA, Schmidt M and Szklo M. Brazilian Longitudinal Study of Adult Health (ELSA-Brasil): Objectives and Design. Am J Epidemiol. 2012;175(4):315-324

8. Autenrieth C, Schneider A, Döring A, Meisinger C, Herder C, Koenig W, Huber G and Thorand B. Association between different domains of physical activity markers of inflammation. Med. Sci. Sports Exerc. 2009:41(9):1706-13.

9. Pate RR, Pratt M, Blair SN, Haskell WL, Macera CA, Bouchard C, Buchner D, Ettinger W, et al.Physical Activity and Public Health: A Recommendation From the Centers for Disease Control and Prevention and the American College of Sports Medicine. JAMA. 1995; 273(5):402-7.

10. Rosenberg DE, Bull FC, Marshall AL, Sallis JF and Bauman AE. Assessment of sedentary behavior with the International Physical Activity Questionnaire. J Phys Act Health. 2008;5(1):30-44.

11. Pate RR, O'Neill JR and Lobelo F. The evolving definition of "sedentary". Exerc Sport Sci Rev. 2008;36(4):173-178

12. Katzmarzyk PT, Church TS, Craig CL and Bouchard C. Sitting time and mortality from all causes, cardiovascular disease, and cancer. Med Sci Sports Exerc. 2009:41(5):998-1005

13. Matsudo VKR e Andrade DR. Aconselhamento sobre Atividade Física. Diagn Tratamento. 2008;13(1):45-9.

14. Zan RA, Silveira L e Zan RP. Prescrição de atividade física: comparação do nível de conhecimento entre alunos da Faculdade de Ciências Médicas da Santa Casa de São Paulo. Anais do XXII Simpósio Internacional de Ciências do Esporte.1999;151.

15. Horita SA, Yamaguti DO and Silveira L. Knowledge level of the new paradigm of recommendation of physical activity for the health among medical students. Med Sci Sports Exerc. 2000;32:313.
16. Frank E, Tong E, Lobelo F, Carrera J and Duperly J. Physical activity levels and counseling practices of U.S. medical students. Med Sci Sports Exerc. 2008;40(3):413-21.

17. Duperly J, Lobelo F, Segura C, Sarmiento F, Herrera D, Sarmiento OL and Frank E. The association between Colombian medical students' healthy personal habits and a positive attitude toward preventive counseling: Cross-sectional analyses. BMC Public Health. 2009; 9:218.

18. Seixas AM, Matsudo SMM, Matsudo, VKR, Andrade EL e Braggion GF. Padrão da prescrição de atividade física realizada por médicos ortopedistas brasileiros. Rev Bras Ciên e Mov. 2003;(11)2:63-9.

19. Matsudo S, Araujo T, Matsudo V, Andrade D, Andrade E, Oliveira LC e Braggion G. Questionário internacional de atividade física. Estudo de validade e reprodutibilidade no Brasil. Rev Bras Ativ Fis Saúde. 2001;(6)2:5-18.

20. Pardini R, Matsudo S, Araújo T, Matsudo V, Andrade E, Braggion G, Andrade D, Oliveira L, Figueira Jr A e Raso V. Validação do Questionário Internacional de Nível de Atividade Física (IPAQ - Versão 6): estudo piloto em adultos jovens brasileiros. Rev Bras Ciên e Mov. 2001;(9) 3:45-51.

21. Haskell WL, Lee I, Pate RR, Kenneth PE, Blair SN, Franklin BA, Macera CA, Heath GW, Thompson PD and Bauman A. Physical activity and public health: Updated recommendation for adults from the American College of Sports Medicine and the American Heart Association. Circulation. 2007;1 16:1081-93.

22. Marcondelli P, Costa THM e Schmitz BAS. Nível de atividade física e hábitos alimentares de universitários do $3^{\circ}$ ao $5^{\circ}$ semestres da área da saúde. Rev Nutr.2008;21(1):39-44.

23. Rodrigues EFR, Cheik NC e Mayer AF. Nível de atividade física e tabagismo em universitários. Rev Saúde Pública.2008:42(4):672-78.

24. Fontes ACD e Vianna RPT. Prevalência e fatores associados ao baixo nível de atividade física entre estudantes universitários de uma universidade pública da região nordeste - Brasil. Rev Bras Epidemiol. 2009;12(1):20-9.

25. Silva GFS, Bergamaschine R, Rosa M, Melo C, Miranda Re Bara Filho M. Avaliação do nível de atividade física de estudantes de graduação das áreas saúde/biológica. Rev Bras Med Esporte. 2007;(13)1:39-42

26. Dubnov-Raz G, Berry EM, Shemer O and Constantini NW. Who will take care of the caretaker? Lifestyle recommendations for physicians. Harefuah. 2011;150(7):583-7.

27. Lobelo F, Duperly J and Frank E. Physical activity habits of doctors and medical students influence their counselling practices. Br J Sports Med. 2009;43(2):89-92.

28. Van der Veer T, Frings-Dresen MH and Sluiter JK. Health behaviors, care needs and attitudes towards self-prescription: a cross-sectional survey among dutch medical students. Plos One 2011;6(11): e28038.

29. Michelucci MA e Matsudo SMM. Grau de conhecimento sobre prescrição da atividade física em três grupos distintos. Anais do XVII Congresso Pan-americano de Medicina do Esporte, 1997.

30. Exercise is Medicine.org [Internet]. American College of Sports Medicine. Disponível em: www.exerciseismedicine.org. Acessado em 14 de Abril de 2012 